\title{
An Overall Review of Linguistic Research on Genre
}

\author{
Guowei Ren \\ Foreign Languages School, Qingdao University of Science and Technology \\ 69 Songling Street, Qingdao 266071, China \\ E-mail: derekxiaoxiao@163.com
}

\begin{abstract}
The essay mainly explores the contemporary linguistic research on genre and makes a brief and preliminary analysis of advantages and disadvantages of influential generic theories. It argues that the orientation for ensuing further generic research should be concentrated on multi-generic texts.
\end{abstract}

Keywords: Genre, Multi-generic texts, Generic analysis

1. Concept of Genre

The concept of genre is currently a widely-discussed research area in linguistics as well as in literature. In literature, there is a long tradition of the study of genres, which can be traced back to the ancient Greek philosopher Aristotle. It is generally accepted that Aristotle was the first scholar to propose the three "natural forms' of poetry: epic, lyric and drama. Among the literary genres, the most well-developed and well-studied genre would most probably be narrative ones, especially the genres of fairy tales and parables. The concept of genre, a word of French origin which means kind or type, was generally restricted to literary works, such as poetry, novels, and dramas. Russian scholar Brahmin was the first researcher who greatly extended the denotation of the concept so as to include non-literary works under its heading, such as classified advertisements, news reports, and scientific essays or papers.

Since the eighties of the twentieth century, more and more linguistic research has been riveted on the study of the unit above the text, namely genre. This trend has drawn the attention from a large variety of linguistic specialists, including socio-linguists, ethnography researchers, anthropologists, rhetoric scholars, language educationalists and even philosophers. There may be two main reasons underlying the motivation of such academic trend. The first is that along with the rise of discourse analysis and textual-linguistics, the focus of linguistic research has gradually shifted from the lower level units---the relatively smaller phonological and lexical-grammatical units such as phonemes, morphemes, words, word groups or phrases, clauses---to some larger units, paragraphs and texts or discourses. Some fundamental issues in the study of text or discourse are closely related to the concept of genre. For example, in the exploration of coherence or cohesion in a text, some linguists have come to know that genre is also an important factor influencing how one part of a text coheres with another or what cohesive devices are selected or fore-grounded in a text. Therefore it is necessary to make an effective investigation of genre in order to get a better understanding of its realization form ---the text. The second motivation is due to the fact that the study of genre has practical uses in language education, especially in second language teaching. The mastery of a particular genre could improve a student's capability to get a thorough comprehension of a text realizing that genre or to compose a good passage.

\section{Linguistic Theories for Generic Analysis}

\subsection{Generic Structure Potential by Hasan}

Systemic functional linguists have made great contributions to generic research. The world renowned linguist Halliday, who is the pioneer of systemic-functional linguistics, has paid attention to generic analysis for a long time. He has made some exploration of the nature and functions of genre in some of his works. In his book Language as Social Semiotic: The Social Interpretation of Language and Meaning, Halliday (1978, pp. 61) argues that 'In order to give a complete characterization of texture, we should have to make reference also to 'generic' structure, the form that a text has as a property of its genre.' Hasan has gone further in the exploration of generic study. In her collaborative work with Halliday Language, context, and text: aspects of language in a social semiotic perspective, She first defines the concept of genre as 'type of discourse', and then proceed to make a much more profound investigation of its structure.(Halliday \& Hassan, 1989, Part B) She proposes a theory for generic analysis: generic structure potential (often abbreviated to GSP. In the following part of the 
thesis, the initial is adopted).Generally speaking, the essence of her GSP theory consists of three main propositions stated as follows:

The features of contextual configuration could be used for making some kind of predictions about text structure and the sequence and order of the elements in the structure. Contextual configuration is composed of three components: field of discourse, tenor of discourse, and mode of discourse, each of which may be thought of as a variable that is represented by some specific values.

Every genre has a generic structure potential, in which there are three kinds of elements: obligatory, optional, and recursive. The generic structure potential for a genre is a linear combination of the above-mentioned three elements in a certain order or sequence.

Genre is defined by obligatory elements in structure. By implication, the obligatory elements and their sequence define the genre to which a text belongs. The function of optional elements may account for the variations in all the texts that belong to the same genre.

Hassan's theory provides a successful explanation for the phenomenon that countless texts whose forms may differ significantly are perceived by the native speakers of that language to have close association with one another. As we know, when required to write an essay on the same subject matter, different person produces different texts. For example, a language teacher gives his pupils an assignment to write a passage about one's most exciting experience in the summer vacation. Each student would produce a text different from other students', but all the products of the students share some similarities in spite of the formal differences. In other words, all the texts belong to one genre, that is, the narrative genre, and they are part of the all possible actual realizations of the generic structure potential for that genre. According to Hassan's GSP theory, all the texts written by students have the same obligatory elements, which account for the textual similarities perceived unconsciously by the native speakers. The textual variations are caused by different choices of optional elements in the generic structure. Hassan is the first systemic-functional linguist who has made a systematic probe into the concept of genre, providing a theoretical basis for further research into the area.

\subsection{Flowchart Theory by Ventola}

Though Hassan's theory provides a reasonable explanation of the commonality and variation among all the texts of a particular genre, it also suffers from certain kind of theoretical weakness. Firstly, the general framework for generic analysis by Hassan is based on a linear sequence, in which all the elements, obligatory or optional, are arranged in a linear structure with one element following another. As Ventola states in her book The Structure of Social Interaction: A Systemic Approach to the Semiotics of Service Encounter, the linear sequence is often troubled with the following problems: (Ventola, 1987, pp. 75-78)

The arrangement of elements in the linear framework does not necessarily accord with the sequence in actual texts.

The recursive or iterative nature of certain element in actual texts is much more complex and complicated than that has been discussed by Hassan.

Some elements in a generic structure may be alternatively realized by some non-linguistic behaviors, rather than linguistic forms.

Secondly, there is an excessively strong association of obligatory elements of text structure with the contextual variable field in Hassan's theory. For Hassan, text structure is the realization of choices made from among the options constituting a culture's field, mode and tenor. In practice, obligatory elements of structure appear to derive from field, with variations in generic structure controlled by tenor and mode. If a genre is defined by its obligatory elements, it follows that their changes in accordance with any alternations in the value of contextual factor field may lead to the generation of numerous new genres.

Thirdly, as Hassan approaches genre analysis from a static, rather than a dynamic, perspective, it encounters some difficulty in accounting for some failures in the process of social interaction, such as a transaction in a market. Take the example text in the genre of everyday service encounter presented by Hassan. If the vendor does not have or has sold out the item of goods demanded by the customer, then the whole interaction may break down without any overall goal accomplished. As a result of this, the text produced under that circumstance may also be called a complete text, but it does not have realized all the obligatory elements in the generic structure of service encounter. GSP theory fails to offer a satisfactory explanation for that case.

In a summary, the formulation of generic structure potential presented by Hasan provides a fundamental basis for genre analysis. At the same time, the theory also has some weakness, which needs to be revised or perfected. In 
the later part of my thesis, I will make further discussion of Hasan's theory and make some revision and perfection to it.

Ventola has followed up the above problems in detail for service encounter genres and developed a flowchart notation for expressing genre as a dynamic potential from which structural elements can be derived. The major distinction between Ventola's flowchart theory and Hassan's GSP formulation consists in the fact that the former approaches genre analysis from a dynamic perspective, rather than from a synoptic perspective. The advantage of the dynamic perspective is that choices can be conditioned by the point reached in a text's development. In the flowchart designed by Ventola, the choice at a certain point is influenced by the option that has been made in the previous part of a text. For example, the lack of the kind of merchandise demanded by the customer may lead to the suspension of a potential transaction. In that case, the choice in Sales Compliance differs from the one made in a successful service exchange interaction, and serves as a presupposed condition which would affected the selection of options in the following elements in the generic structure. Therefore, the flowchart theory offers a satisfactory explanation about the failures in a social interaction such as service encounter. As Martin comments on Ventola's model of analysis, 'not only is this work (the flowchart theory) the most detailed study of generic structure within a systemic functional framework but it complements in critical ways the synoptic orientation of work inspired by Hassan and Martin'. (Martin, 1992, Chapter 7) However, in her framework of analysis, the notion of text is positioned as a combined plane of content and expression, which is the realization of both register and genre in language. It is obvious that her definition of texts is in contradiction with that of Halliday, who always stresses that 'a text is a unit of meaning.' (Halliday, 1978, pp. 60)

\subsection{Schematic Structure Proposed by J.R. Martin}

Another systemic-functional linguist who has made profound and innovative research into genre analysis is the Australian scholar J.R. Martin. He (1992, Chapter 7) argues that in the interpretation of context there are four communicative planes: ideology, genre, register, and language. Ideology in combination with genre should be identified as two planes of context of culture. Ideology is a system of coding orientations which makes meaning selectively available depending on subjects' class, gender, ethnicity and generation. It exerts a certain extent of restrictive influence on the choice of genres, which functions as its expression form as well as register, and language. In contrast to Hassan's GSP theory, text structure is referred to as schematic structure in Martin's model. The term schematic simply refers to the staged, step-by-step organization of the genre, or, in Martin's terms, represents the positive contribution genre makes to a text: a way of getting from A to B in the way a given culture accomplishes whatever the genre in question in functioning to do in that culture.

A comparison of these two models of Hassan and Martin would show that they have much in common, for both of them stress the strong correlation between the sequence of elements or stages and field, mode and tenor options. Their major difference lies in the fact that Hassan's model makes a distinction between obligatory elements and optional elements, whereas Martin does not make such a distinction. On the issue of the necessity to establish another separate communicative plane ideology, the scholars within the systemic-linguistics could not achieve a common consensus. When talking about the relationship between genre, register and language, Eggins claims that 'genre is one of the two levels of context we are recognizing'. (Eggins, 1994, Chapter 2) In other words, Eggins identifies genre with the more general and abstract context---context of culture as the same thing.

\subsection{Communicative Event Proposed by Swales}

The above discussion is mainly focused on the research work in systemic-functional camp. However, that is just part of the whole story, and there is another linguist outside the systemic functional linguistic circle who has invested such outstanding efforts in the area that it is worthwhile to give a brief introduction to his research results here. That is John M. Swales in the University of Michigan, the USA. In his work Genre Analysis: English in Academic and Research Settings, Swales (1990, pp.33-61) argues that a genre comprises a class of communicative events, the members of which share some set of communicative purposes. According to the point of view of Swales, a communicative event is assumed to be one in which language plays a significant and an indispensable role, and the activities in which talk is incidental, such as engaging in physical exercise, doing the household chores, or driving, will not be considered as communicative events. In other words, only those linguistic activities or activities in which linguistic behaviors predominate can be regarded as communicative events; Non-linguistic activities are not included in this category. The principal criterion that turns a collection of communicative events into a genre is some shared set of communicative purposes. The lack of an overall purpose in any verbal activities would exclude them as instances of genres. Thus, the following two areas of verbal activity are considered to lay outsides genres: casual conversation or 'chat' and 'ordinary' narrative. Swales' placing the primary determinant of genre-membership on shared purpose rather than on similarities of 
form or some other criterion is to take a position that accords with that of Martin or Eggins. However, his relatively narrow perspective on the concept of genre, especially his exclusion of casual conversation and ordinary narratives as genres, is somewhat at variance with that position held by most systemic-functional linguists.

\section{Common Deficiency in the Contemporary Study of Genre and Orientation for Further Research}

In the previous section, the discussion has been focused on the current state of genre studies, linguistic or literary, systemic-functional or non-systemic-functional. Although those studies have produced a lot of outstanding achievements, there is a common deficiency or weakness in their research: excessive attentions have been concentrated on texts realizing only one genre, whereas the texts which are generated by more than one genre have been paid little attention in their respective research spheres. Both of the two texts employed by Hassan to illustrate her generic structure potential theory are realization examples of only one genre - the genre of service encounter. Among the four texts listed in the second chapter of An Introduction to Systemic-Functional Linguistics by Eggins (1994, Chapter 2), two belong to the transactional genre; the other two the genre of placing a bet and recipe. Although in that book the concept of macro-genre is mentioned, the author does not unfold detailed explanatory paragraphs on that subject. Similarly, in his works Swales does not make any elaboration on the academic writings involved with the realization of more than one genre.

The above-mentioned scholars' ignorance of texts generated by more than one genre does not suggest that there is only a low percentage of that type of texts in the ocean of world's existing literature. On the contrary, the combination of two or more genres in a single text is an extremely universal phenomenon, and its occurrence is much more universal and commonplace than what we can imagine.

There is a number of evidence that could prove the universality of multi-generic phenomenon. In the first place, when we makes a narration about one of our interesting experiences, sometimes we may feel the need to make a kind of description of the characters or figures involved in that experience. From the rhetorical point of view, the insertion of description would make the narration much more vivid and attractive. In the same way it is inevitable that an argumentative writing may consist of some stretches of explanatory paragraphs for the purpose of increasing its persuasive forces. The texts produced by the combination of narration and description, or argumentation and explanation are what we called multi-generic texts.

The second evidence is suggested by the fact that in some longer texts, both spoken and written, a range of genres may be identified within it. For example, a university department's handbook is itself an example of a genre, but it typically contains sections exemplifying the genres of exposition (why a student should study the subject, what it is about), description (course outlines) and regulation (rights/responsibilities/penalties).

Another apparent example of multi-generic phenomenon is the novel. It is generally recognized that in a novel the author could make use of a lot of other kinds of literary genres, such as poetry, letters, and songs. Generally speaking, there is no limit on the type and number of other genres that could be included in a novel; almost all the other genres have the access to be embedded in a novel. It is due to this peculiar character that the novel sometimes is referred to as a royal genre.

In summary, multi-generic texts should be highlighted in the subsequent linguistic research on genre and an appropriate theoretical analytic framework need to be formulated.

\section{References}

Eggins, Suzanne. (1994). An Introduction to Systemic Functional Linguistics. London: Pinter Publishers, (Chapter 2).

Halliday, M.A.K. \& Hasan, Ruqaiya. (1989). Language, Context, and Text: Aspects of Language in a Social-Semiotic Perspective. London: Oxford University Press, (Part B).

Halliday, M.A.K. (1978). Language as Social Semiotic: The Social Interpretation of Meaning. London: Edward Arnold, pp. 60-61.

Martin, J.R. (1992). English Text: System and Structure. Amsterdam: John Benjamins Publishing Company, (Chapter 7).

Swales, J.M. (1990). Genre Analysis: English in Academic and Research Settings. London: Cambridge University Press, pp. 33-61.

Ventola, Eija. (1987). The Structure of Social Interaction: A Systemic Approach to the Semiotics of Service Encounter. London: Frances Pinter, pp. 75-78. 